\title{
Shaping perceptual learning of synthetic speech through feedback
}

\author{
Matthew I. Lehet ${ }^{1} \cdot$ Kimberly M. Fenn ${ }^{1} \cdot$ Howard C. Nusbaum ${ }^{2,3}$ \\ Published online: 4 June 2020 \\ (C) The Psychonomic Society, Inc. 2020
}

\begin{abstract}
Listeners exposed to accented speech must adjust how they map between acoustic features and lexical representations such as phonetic categories. A robust form of this adaptive perceptual learning is learning to perceive synthetic speech where the connections between acoustic features and phonetic categories must be updated. Both implicit learning through mere exposure and explicit learning through directed feedback have previously been shown to produce this type of adaptive learning. The present study crosses implicit exposure and explicit feedback with the presence or absence of a written identification task. We show that simple exposure produces some learning, but explicit feedback produces substantially stronger learning, whereas requiring written identification did not measurably affect learning. These results suggest that explicit feedback guides learning of new mappings between acoustic patterns and known phonetic categories. We discuss mechanisms that may support learning via implicit exposure.
\end{abstract}

Keywords Language comprehension $\cdot$ Implicit vs. explicit memory $\cdot$ Perceptual learning $\cdot$ Psycholinguistics

\section{Introduction}

Native language acquisition is not thought of as explicitly instructed, yet children learn mappings between acoustic features in the speech signal and lexical representations (Idemaru \& Holt, 2013; Nittrouer, Lowenstein, \& Packer, 2009). There is longstanding debate over whether these mappings are acquired through simple exposure, suggesting an innate propensity to acquire spoken language (e.g., Chomsky, 1965; Pinker, 1994), or whether some feedback is necessarily part of acquisition (e.g., Demetras, Post, \& Snow, 1986). Various studies suggest that adults use information in their speech environment to adjust mappings between acoustics and lexical representations, allowing dynamic on-line adjustments to speech categories (Guediche, Fiez, \& Holt, 2016; Idemaru \& Holt, 2014; Kleinschmidt \& Jaeger, 2015; Kraljic \& Samuel, 2006; Vroomen, van Linden, de Gelder, \& Bertelson, 2007).

Matthew I. Lehet

lehetmat@msu.edu

1 Department of Psychology, Michigan State Universit, East Lansing, MI, USA

2 Department of Psychology, The University of Chicago, Chicago, IL, USA

3 Center for Cognitive and Social Neuroscience, The University of Chicago, Chicago, IL, USA
However, the ways in which internally generated predictions and externally provided feedback influence perceptual learning remain unclear.

Across a variety of paradigms, environmental information helps listeners adjust mappings between acoustic features and lexical representations. This learning can happen explicitly through orthographic feedback that informs the mapping between distorted acoustics and lexical representations (Davis, Johnsrude, Hervais-Adelman, Taylor, \& McGettigan, 2005; Fenn, Margoliash, \& Nusbaum, 2013; Guediche et al., 2016; Hervais-Adelman, Davis, Johnsrude, \& Carlyon, 2008; Loebach, Pisoni, \& Svirsky, 2010). However, incidental experience - such as simple exposure (Clarke \& Garrett, 2004; Davis et al., 2005) or listening to speech with systematic regularities between multiple acoustic features (Idemaru \& Holt, 2014; Liu \& Holt, 2015) - results in implicit learning. Speech environments also provide information that is neither explicit nor implicit that can produce learning. Lexical frames (e.g., Kraljic \& Samuel, 2006; Norris, McQueen, \& Cutler, 2003) or concurrent visual information such as speaking faces (e.g., Keetels, Pecoraro, \& Vroomen, 2015; Vroomen et al., 2007) also produce learning. Thus, environmental cues may serve as teaching signals to guide perceptual learning (Guediche, Blumstein, Fiez, \& Holt, 2014) and these teaching signals may range from explicit, such as orthographic feedback, to implicit, such as internally generated hypotheses during passive listening.

Evidence from noise-distorted speech perception suggests that internally generated hypotheses may be important 
because perceptual learning is related to the extent that listeners determine accurate lexical representations (Davis et al., 2005). Interestingly, learning may depend on successfully identifying targets during training, regardless of feedback. Participants who more accurately identified noisedistorted words during training showed more learning from pretest to post-test, regardless of explicit feedback (Guediche et al., 2016). Thus, having a correct lexical target - either based on internal hypotheses or explicit feedback, is similar to hearing more intelligible speech, which is known to improve learning (Bradlow \& Bent, 2008; Clarke \& Garrett, 2004; Peelle \& Wingfield, 2005). Therefore, the perceptual system can adjust acoustic-lexical mappings based on either internally or externally generated targets, which guide learning.

One mechanism by which targets may be used to adjust acoustic-lexical mappings is by minimizing prediction error. In the case of noise-distorted speech, error between expectations and acoustics makes mapping from acoustics to lexical representations difficult. Either explicit feedback or internally generated hypotheses could guide the reduction in prediction error (e.g., Sohoglu \& Davis, 2016). Perceptual learning would result as mappings between acoustics and the lexicon adjust so the acoustics of degraded speech now map onto the correct lexical or phonetic target. Theoretical models of speech perception suggest that deviations between predicted mappings and experience guide learning when targets inform the minimization of prediction error (Kleinschmidt \& Jaeger, 2015; Kuperberg \& Jaeger, 2015). Explicit feedback provides a robust guide for adjusting acoustic-lexical mappings during perceptual learning. Internally generated hypotheses may also provide useful internal teaching signals if correct; however, this has been less thoroughly investigated.

Perceptual learning of early synthetic speech (as in Nusbaum \& Pisoni, 1985) is an ideal paradigm for comparing implicit and explicit learning. Synthetic speech used in this work provides a distorted speech signal in which some acoustic cues are misleading, and some are incorrect, based on American English phonology. When learning to understand synthetic speech, listeners must recognize new acoustic patterns as known phonetic categories, learning new acoustic-phonetic mappings. After training with feedback, adult listeners show significant, longterm improvements in recognizing synthetic speech, and this learning generalizes to novel stimuli (Fenn et al., 2013; Fenn, Nusbaum, \& Margoliash, 2003; Francis, Baldwin, \& Nusbaum, 2000; Francis, Nusbaum, \& Fenn, 2007; Greenspan, Nusbaum, \& Pisoni, 1988; Schwab, Nusbaum, \& Pisoni, 1985). Control conditions with no training or explicit feedback also demonstrated some learning between pretest and post-test simply based on exposure to the pretest speech (see Schwab et al., 1985). Learning was substantially reduced compared to training with explicit feedback, albeit perhaps due to a vastly reduced amount of exposure.
Thus, training with explicit feedback facilitates learning new acoustic-phonetic information about known phonetic categories, but it is unclear whether internal hypotheses could also support learning to the same degree. Studies on perceptual learning of synthetic speech typically involve identification in addition to feedback (e.g., Francis et al., 2000; Greenspan et al., 1988; Schwab et al., 1985). Listeners hear a stimulus, identify it, and receive feedback about stimulus identity. Is this identification necessary for learning? Evidence suggests that internally generated hypotheses about lexical identity assist the generation of internal teaching signals (e.g., Guediche et al., 2016) and facilitate perceptual learning; however, it is unclear how formalizing hypotheses in responses might affect learning.

The present study crossed orthographic feedback (vs. no feedback) and explicit identification (vs. exposure) in a synthetic speech learning task. Training varied according to whether listeners were given explicit word-identity orthographic feedback and whether they identified each word. We predicted that both feedback and identification would benefit perceptual learning relative to a control group that received no training. In Experiment 1, we examine how these processes play out over multiple days of training. In Experiment 2, we examine these processes in a single training session with the addition of confidence judgments on each training trial to further identify how internal predictions are related to perceptual learning.

\section{Experiment 1}

\section{Method}

Participants Seventy-five students or employees from the University of Chicago (mean age $=21.5$ years, $S D=3.4$ years) volunteered for this study. All participants were right-handed, native English speakers, with minimal previous exposure to synthetic speech and no history of hearing or speech disorders. Participants were randomly assigned to one of five groups: four experimental, one control. Informed consent was obtained before participation and participants were compensated US\$10 for each session.

Materials 650 monosyllabic words were generated using rsynth, a text-to-speech synthesizer that generates acoustic parameters based on the Klatt synthesizer (Klatt, 1980). The words were taken from lists of phonetically balanced words (Egan, 1948), approximating the distribution of phonemes in English. The two tests consisted of 100 different words, and the three training sets consisted of 150 different words each. Each word was presented only one time throughout the experiment so participants never heard the same word more than once; thus, all learning was generalized. There were two 
separate 100-word test sets counterbalanced across participants; half of the participants within each group received one set for the pretest and the other set for the post-test, and the other half received the opposite order. The stimuli were presented at a comfortable listening level through Sennheiser HD 570 headphones with an average RMS amplitude of 66.5 dB.

Design Each of the four training groups participated in three experimental sessions and the control group participated in two sessions. The training groups ( $\mathrm{n}=15$ in each condition) received a pretest and one training session on the first day, a second training session on the second day, and a final training session followed by a post-test on the third day. The control group $(n=15)$ received a pretest on the first day and a post-test 2 days later, with no training. The training groups differed based on a $2 \times 2$ design that crossed presence or absence of orthographic feedback with whether or not explicit stimulus identification was required on each training trial. Feedback was the orthographic form of the word printed on the computer screen (i.e., word-identity feedback), paired with auditory presentation of the stimulus. The orthographic feedback and auditory stimulus were presented at the same time. However, due to the temporal nature of the auditory signal, the printed orthography was available before the full auditory signal. In the no-feedback condition, participants received only auditory presentation of the stimulus. Half of the participants in each condition were asked to explicitly identify the stimuli (ID groups).

Procedure During the pretest and post-test, participants heard 100 individual words and identified each stimulus by typing the word they heard. There was no time limit on responses and no feedback during the tests.

Training groups were presented with 150 different synthetic speech words during each of the three training sessions, for a total of 450 unique training words. On each trial, an auditory stimulus was presented through headphones. After the auditory presentation, ID group participants were given $4 \mathrm{~s}$ to identify the word by typing their response on the keyboard. After this, half of the ID participants received a second auditory presentation of the word alone and the other half received an auditory presentation of the word paired with the orthographic form of the word. For the two groups that were not required to identify the stimuli, training words were presented individually with a 1,500-ms inter-stimulus interval, either with wordlevel orthographic feedback, or alone. Thus, on trials that required identification, participants heard two repetitions of each word, whereas when identification was not required, participants heard only one presentation. All training sessions were divided into three sets of 50 trials. At the conclusion of 50 trials, subjects were reminded of their task and given a short break.

\section{Results: Testing}

Testing and training data were scored as percent correct word identification. A response was considered correct if every phoneme was correctly transcribed whether spelled correctly or not. Unexpectedly, there were small but significant differences in pretest performance (see Table 1, $\mathrm{F}_{4,70}=2.63, p=.04$ ). Given counterbalancing of test forms, the differences between the groups were due to participant sample variation reflecting individual differences and not test effects. There was a trend for the two groups that identified the stimuli to show higher pretest accuracy than the other groups (Table 1), but a post hoc Sheffe's test found that none of the individual contrasts were significant ( $p>.2$ for all). Because initial performance differed slightly among groups, we assessed the relationship between baseline performance (pretest accuracy) and learning (performance increase from pretest to post-test) across our entire sample and found that there was not a significant correlation, $r=.14, p>.2$.

Although pretest performance did not predict learning, we conducted all analyses on the amount of learning as measured by the percentage point difference between pretest and posttest performance, to control for baseline differences. The amount of learning varied by condition (see Table 2, $\mathrm{F}_{4,70}=38.8, p<.001$ ), and planned contrasts suggest that all four training groups displayed greater learning than the control group ( $\mathrm{t}_{28}>2.8, p<.01$ for all comparisons). Moreover, the control group did not improve reliably from pretest to posttest, (see Table 1, $\mathrm{t}_{14}=.66, p=.52$ ).

For the four experimental groups, the magnitude of learning varied across training conditions. Experimental group data were analyzed using a two-factor ANOVA with orthographic feedback and identification as between-subjects factors. Figure 1 shows performance for the two groups that received orthographic feedback increased an average of $29.3 \pm 1.7$ and $25.8 \pm 1.6$ (mean \pm s.e.m.) percentage points from pretest to post-test (with and without identification, respectively), which was significantly greater than the two groups that received only auditory exposure. These auditory exposure groups increased only $10.1 \pm 3.7$ and $8.6 \pm 2.1$ from pretest to post-test (with and without identification, respectively). Thus, the effect of orthographic feedback on the amount of learning was significant $\left(\mathrm{F}_{1,56}=77.1, p<.001\right)$. However, word identification did not significantly affect learning $\left(\mathrm{F}_{1,56}=1.5, p=.23\right)$, even though participants heard each stimulus twice during identification trials and therefore had double the stimulus exposure compared with the non-identification groups. There was not a significant interaction between the factors of feedback and identification $\left(\mathrm{F}_{1,56}=.23, p=.63\right)$.

These results demonstrate that exposure to synthetic speech is sufficient to produce significant generalized learning. In contrast, neither doubling the amount of exposure to the same words nor an identification task increased the amount of learning. However, word-identity feedback significantly enhanced learning. 
Table 1 Average percent correct (and standard error of the mean.) word identification at pretest, post-test, and the amount of learning between pretest and post-test for each of the five groups in Experiment 1 and the four groups in Experiment 2

\begin{tabular}{|c|c|c|c|}
\hline & Pretest & Post-test & Learning \\
\hline \multicolumn{4}{|l|}{ Experiment 1} \\
\hline Control & $31.5(3.0)$ & $32.4(2.8)$ & $0.9(1.3)$ \\
\hline Auditory exposure & $31.6(1.6)$ & $40.2(2.9)$ & $8.6(2.1)$ \\
\hline Auditory exposure and orthographic feedback & $39.3(2.0)$ & $65.1(2.4)$ & $25.8(1.6)$ \\
\hline Identification with auditory exposure & $37.3(2.2)$ & $47.4(4.2)$ & $10.1(2.7)$ \\
\hline Identification with auditory exposure and orthographic feedback & $32.0(2.3)$ & $61.3(2.9)$ & $29.3(1.7)$ \\
\hline \multicolumn{4}{|l|}{ Experiment 2} \\
\hline Auditory exposure & $20.1(0.8)$ & $23.2(1.4)$ & $3.1(1.2)$ \\
\hline Auditory exposure and orthographic feedback & $20.1(1.1)$ & $29.7(1.5)$ & $9.6(1.4)$ \\
\hline Identification with auditory exposure & $21.4(0.9)$ & $28.2(1.5)$ & $6.8(1.2)$ \\
\hline Identification with auditory exposure and orthographic feedback & $20.3(1.0)$ & $31.2(1.4)$ & $10.8(1.3)$ \\
\hline
\end{tabular}

\section{Results: Training}

Although all four experimental groups received training, we did not collect responses for the groups that listened passively to the stimuli. Therefore, training scores were examined only for the two groups that identified the stimuli during training.

Data from the training sessions was analyzed as a two-factor ANOVA with training session as a repeated measure and feedback as a between-subjects factor. Figure 2 demonstrates a reliable effect of training (also see Table $2, \mathrm{~F}_{2,56}=74.97, p<.001$ ), with performance improving on each subsequent session. There was also a significant effect of condition $\left(\mathrm{F}_{1,28}=5.2, p<.03\right)$. The orthographic feedback group performed better than the auditory repetition-only group (Table 2). Perhaps more interesting, there was also a significant interaction between condition and training session $\left(\mathrm{F}_{2,56}=16.97, p<.001\right)$, which suggests that the orthographic feedback group both performed better overall compared to the auditory repetition only group, and also improved more at each training session. Thus, even though both groups improved throughout training, the magnitude of the improvement was greater in the group that received orthographic feedback on word identity.

\section{Experiment 2}

The second experiment was designed to replicate Experiment 1 and further investigate how internal predictions are related to
Table 2 Average percent correct (and standard error of the mean, s.e.m.) word identification during the three training sessions for the group that was given auditory exposure alone after identification and the group that was given orthographic feedback after identification in Experiment 1. Average percent correct (and s.e.m.) word identification

\begin{tabular}{|c|c|c|c|}
\hline Experiment 1 Accuracy & Session 1 & Session 2 & Session 3 \\
\hline Identification with auditory exposure & $36.5(2.4)$ & $39.7(3.2)$ & $43.3(2.9)$ \\
\hline Identification with auditory exposure and orthographic feedback & $38.9(2.7)$ & $47.8(2.6)$ & $58.1(2.7)$ \\
\hline Experiment 2 Accuracy & Block 1 & Block 2 & Block 3 \\
\hline Identification with auditory exposure & $25.8(1.1)$ & $27.4(1.5)$ & $29.3(1.5)$ \\
\hline Identification with auditory exposure and orthographic feedback & $25.7(1.3)$ & $30.5(1.3)$ & $32.6(1.5)$ \\
\hline Experiment 2 Confidence & Block 1 & Block 2 & Block 3 \\
\hline Auditory exposure & $3.30(0.19)$ & $3.30(0.21)$ & $3.25(0.21)$ \\
\hline Auditory exposure and orthographic feedback & $3.74(0.15)$ & $3.87(0.16)$ & $3.89(0.19)$ \\
\hline Identification with auditory exposure & $3.52(0.17)$ & $3.53(0.18)$ & $3.59(0.19)$ \\
\hline Identification with auditory exposure and orthographic feedback & $3.66(0.15)$ & $3.72(0.17)$ & $3.73(0.18)$ \\
\hline
\end{tabular}

during the three 100-trial training blocks for the group that was given auditory exposure after identification and the group that was given orthographic feedback after identification in Experiment 2. Average confidence ratings (and s.e.m.) for all four groups in Experiment 2 


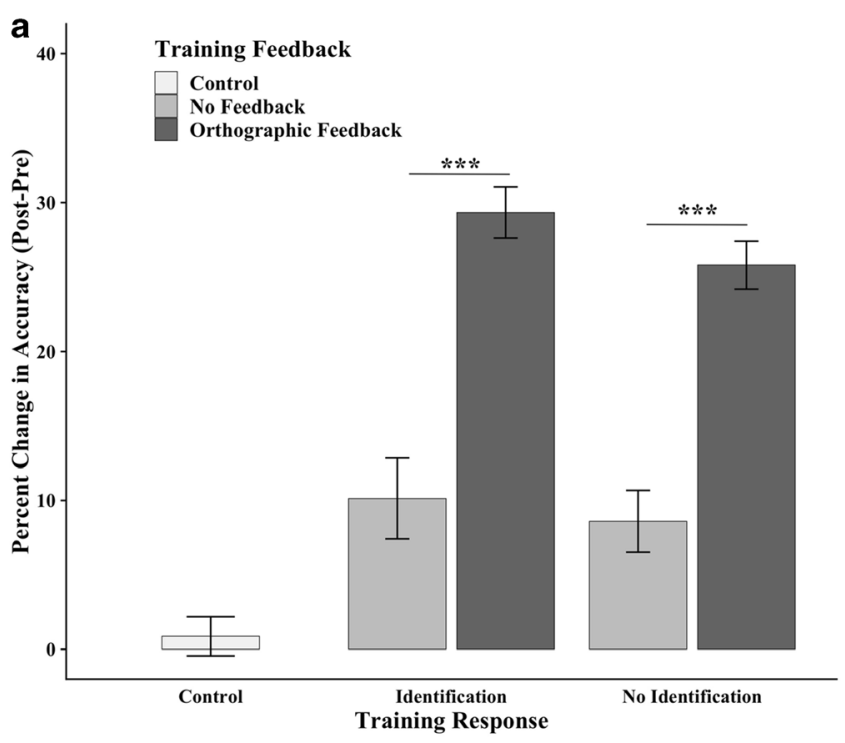

Fig. 1 (A) Improvement, or change in percent correct word identification accuracy from pretest to post-test [(percent correct $\left._{\text {post-test }}\right)-$ (percent correct $\left.t_{\text {pretest }}\right)$ ], across groups that received orthographic feedback versus no feedback, when identification through written responses was or was not required during training. The control group experienced no training. Error bars represent standard error of the mean. (B) Average percent

learning. To this end, we added confidence judgments on each training trial for all groups.

\section{Methods}

Participants A total of 137 students from Michigan State University (mean age $=19.0$ years, $S D=1.1$ years) volunteered for this study. All participants were right-handed, native English speakers, with minimal previous exposure to synthetic speech and no history of hearing or speech disorders. Participants were randomly assigned to one of four experimental groups. All participants provided informed consent and were compensated with course credit. Four participants were excluded for failing to respond on the pretest, training, or post-test.

Materials The same stimuli were used as in Experiment 1. The pretest and post-test were the same, but training consisted of only 300 words. Each word was presented only one time throughout the experiment so participants never heard the same word more than once; thus, all learning was generalized. Test lists were counterbalanced across participants. The stimuli were presented at a comfortable listening level.

Design Each of the four groups participated in a single session. Training crossed presence or absence of orthographic feedback with whether or not explicit stimulus identification was required on each training trial. Unlike Experiment 1, on each trial, participants rated how confident they were that they

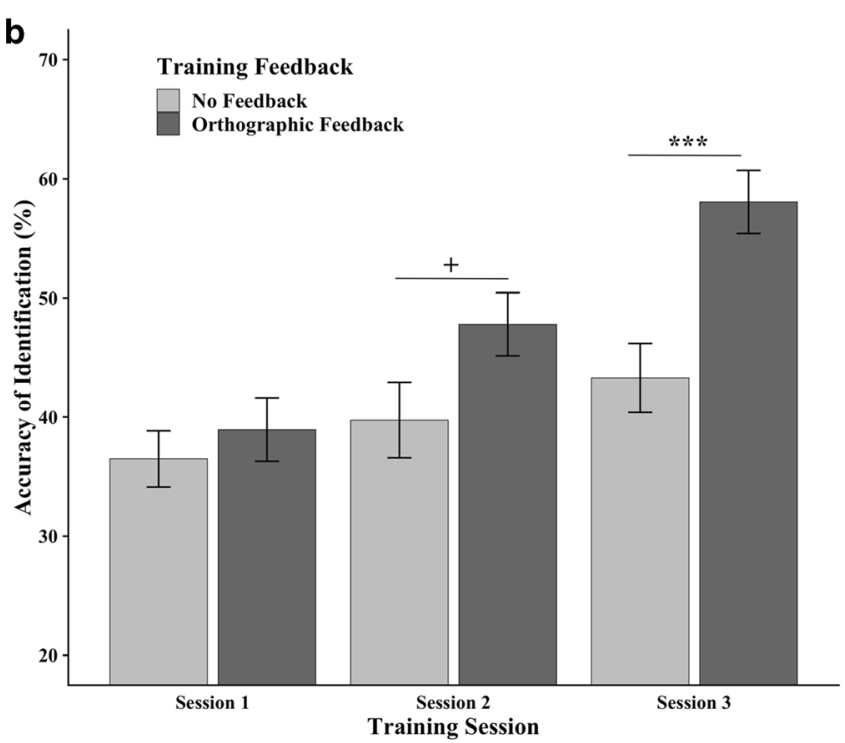

correct for the 150 words during the first training session (on day 1 of the study), second training session (on day 2 of the study), and the third training session (on day 3 of the study) for the two groups that provided identification on training trials that either received orthographic feedback or no feedback. Error bars across plots reflect standard error and significance is indicated as $+\mathrm{p}<0.1 ; * \mathrm{p}<.05 ; * * \mathrm{p}<.01 ; * * * \mathrm{p}<.001$

understood the word correctly on a scale from $1=$ Not at all confident to $7=$ Extremely confident. Feedback and identification instructions were identical to Experiment 1. Training occurred immediately after the pretest and before the post-test within the single session.

Procedure The pretest and post-test were identical to Experiment 1 with neither feedback nor confidence ratings.

During training, participants were presented with 300 unique synthetic speech words. On each trial, an auditory stimulus was presented and participants provided a confidence rating. Then, ID groups were given $4 \mathrm{~s}$ to identify the word by typing their response on the keyboard. Finally, the auditory stimulus was played again (for all participants) and for the feedback groups this was paired with the orthographic form of the word. At the conclusion of 50 trials, subjects were reminded of their task and given a short break.

\section{Results: Testing}

Unlike in Experiment 1, there were no baseline differences in the pretest performance between groups $\mathrm{F}_{3,129}=.44, p=.793$. We used the same metric of change in identification accuracy between pretest and post-test to assess learning. We conducted a two-factor ANOVA with orthographic feedback and identification as between-subjects factors. Figure 2 shows that performance in the two groups that received orthographic feedback increased an average of $10.8 \pm 1.3$ and $9.6 \pm 1.4$ percentage points from pretest to post-test (with and without identification, 
a

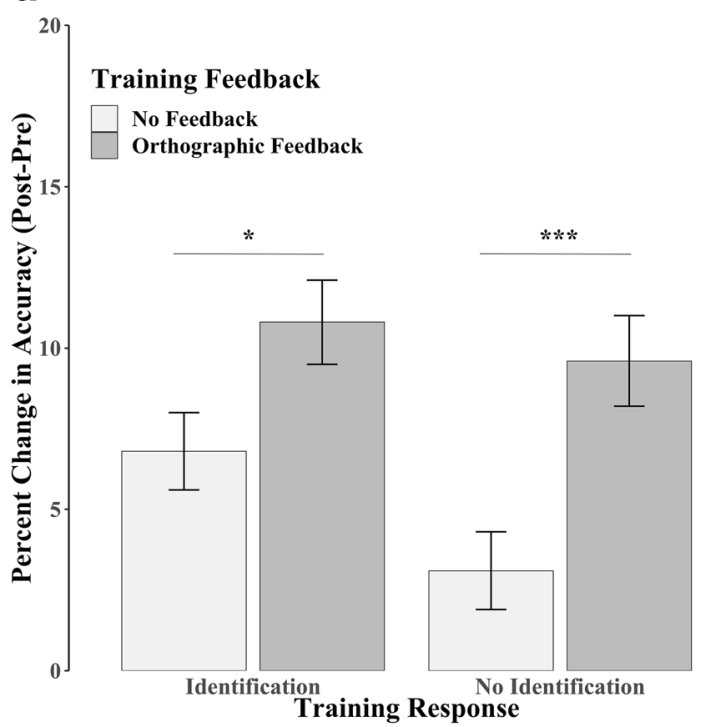

b

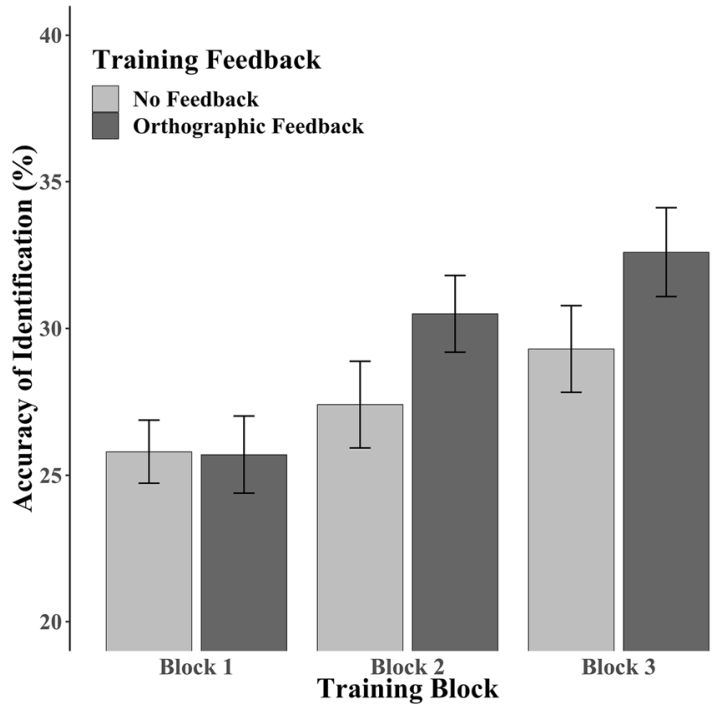

C

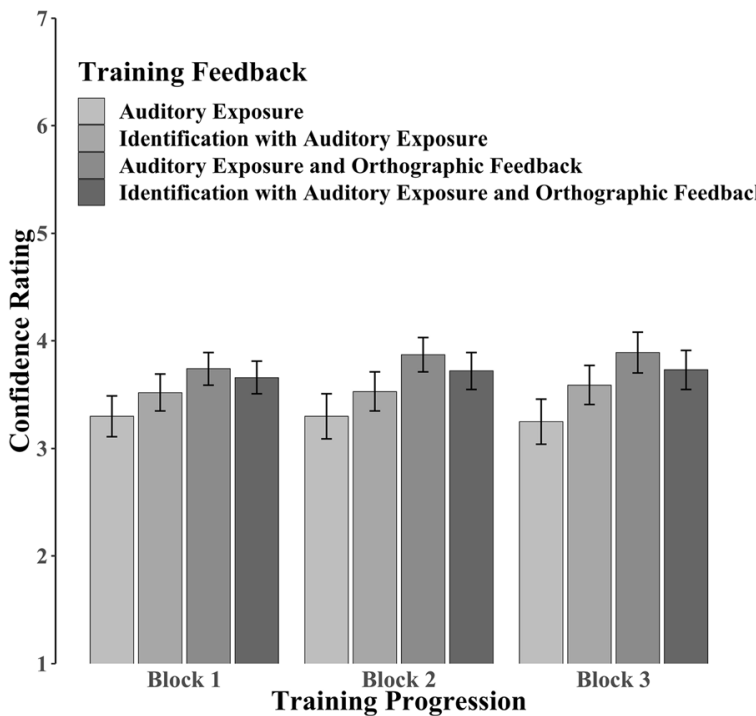

Fig. 2 (A) Change in percent correct word identification accuracy from pretest to post-test across groups that received orthographic feedback versus no feedback, when identification through written responses was or was not required during training. Error bars represent standard error of the mean. (B) Average percent correct for the 100 words in the first, second, and third blocks of training trials for the two groups that provided identification on training trials that either received orthographic feedback or no feedback. (C) Average confidence ratings for the 100 words in the first, second, and third blocks of training trials for all four groups in Experiment 2. Error bars across plots reflect standard error and significance is indicated as $+\mathrm{p}<0.1 ; * \mathrm{p}<.05 ; * * \mathrm{p}<.01 ; * * * \mathrm{p}<.001$

respectively), which was significantly greater than the two groups that received only auditory exposure, who increased only $6.8 \pm 1.2$ and $3.1 \pm 1.2$ from pretest to post-test (with and without identification, respectively). Thus, the effect of orthographic feedback was significant $\left(\mathrm{F}_{1,129}=17.04, p<.001\right)$. However, word identification did not significantly affect learning $\left(\mathrm{F}_{1,129}=3.67, p=.058\right)$ and there was not a significant interaction between feedback and identification $\left(\mathrm{F}_{1,129}=0.90\right.$, $p=.345$ ). These results largely replicate Experiment 1 .

\section{Results: Training}

Figure 2 demonstrates a reliable effect of training block on accuracy (also see Table 2, $\mathrm{F}_{2,128}=21.81, p<.001$ ), with performance improving on each block of 100 trials. Unlike in Experiment 1, there was not a significant effect of condition on training accuracy $\left(\mathrm{F}_{1,64}=1.45, p=.234\right)$ but there was a significant interaction between condition and training block $\left(\mathrm{F}_{2,128}=3.08\right.$, $p=.049$ ), suggesting that the orthographic feedback group improved more across the blocks within the training session.

The addition of confidence trials in the training for all groups allowed us to address how confidence in perception changes as a function of training condition. Figure 2 and Table 2 present these results. We conducted a repeated-measures ANOVA with block as a repeated measure and identification task condition and feedback condition as between-subjects factors. This analysis revealed that orthographic feedback significantly impacted confidence $\left(\mathrm{F}_{1,129}=4.23, p=.042\right)$ but no other effects were significant (all $\mathrm{F}$ statistics $<1.29, p$ values $>.266$ ).

\section{Discussion}

One view of perceptual learning unifies both implicit and explicit learning as the same process albeit with different ways of orienting attention to stimulus information (e.g., Seitz \& Watanabe, 2005). By this view, explicit feedback directs attention to the to-be-learned properties. In incidental learning, internally generated signals should do the same. The present experiments directly compared the role of word identity hypotheses with external orthographic feedback, and demonstrated that in the absence of explicit feedback, word identification 
does not support learning. The finding that the identification task does not improve accuracy relative to exposure suggests that the hypotheses participants are asked to formalize are most likely similar to the guesses that participants simply listening are also making. Therefore, externalizing these guesses in the identification task does not provide a benefit to learning. The results also show that learning occurs with incidental auditory exposure; participants who passively listened improved significantly from pretest to post-test. However, improvement was significantly greater when auditory exposure was coupled with orthographic feedback. Importantly, all learning reflects generalized learning rather than rote memorization. These results align with recent theoretical frameworks that suggest prediction error between expectations and perceived acoustics can tune mappings between acoustic features and linguistic categories, and elaborate upon them, suggesting that requiring predictions through identification responses does not render such prediction error more efficacious.

Theoretical frameworks suggest that prediction error guides perceptual speech learning. Connectionist models could explain these results because accurate target information obtained from orthographic feedback allows the system to adjust weightings (instantiated as connection weights - see Liu \& Holt, 2015) between acoustic input and phonetic categories (Magnuson, Mirman, \& Harris, 2011; McClelland, Mirman, \& Holt, 2006; Mirman, McClelland, Holt, \& Magnuson, 2008). Furthermore, connectionist models can predict reduced learning without feedback. Initially, activation patterns from synthetic speech may partially activate some phonetic representations. Given limited lexical options, if enough correct phonemes are activated, changes to acousticphonetic weightings could produce learning even without orthographic feedback. Further, these results challenge models that suggest feedback plays no role in speech recognition (e.g., Norris, McQueen, \& Cutler, 2000).

Bayesian models could also explain our results. These models suggest that prior probability distributions characterize the likelihood of hearing a phoneme given perceived acoustic features. These probability distributions guide inference decisions of which phoneme was heard (Brown \& Kuperberg, 2015; Kleinschmidt \& Jaeger, 2015; Kuperberg \& Jaeger, 2015). However, priors for synthetic speech are initially unknown. Feedback provides reliable targets to create and guide changes in prior probability distributions but it is unclear how such priors are generated without feedback - yet, learning occurs through exposure. Internal hypotheses may provide such initial priors; however, without feedback, incorrect decisions about the target towards which mappings should be adjusted could be erroneously inferred, causing inaccurate adjustment of priors. Lexical level generalization could occur through adjustments of phonetic priors, allowing phonetic inferences to generate lexical level priors - and inferences even to novel words.
Across these frameworks is a shared suggestion that perceptual learning affects the mappings between acoustics and phonemes, supported by the observation that learning specific lexical items would not provide the generalized benefit seen here. The results further suggest that minimization of prediction error does not depend upon reporting predictions - given that exposure alone demonstrates comparable learning to exposure plus identification in Experiment 1. It is notable that there are small differences in Experiment 2 between exposureonly and exposure-plus-identification conditions in confidence measures during training and in learning. These differences may suggest that written responses result in different underlying cognitive processes than those required for a confidence judgment such as increasing the precision of Bayesian priors or the strength of the target that propagates error-related signals through a connectionist network. Overall though, the results suggest that the predictions most relevant to learning are likely internal to the perceptual system and that they are similar whether reported or not. Clearly, the effect of feedback overrides any task-related identification effect, and so explicit top-down knowledge must also modify these predictions.

Although Experiment 2 largely replicated Experiment 1, there were some small differences between experiments. Primarily, in Experiment 2, which was conducted in a single session, there was not a main effect of condition during training. There are several possible explanations for this discrepancy. Perhaps the most parsimonious explanation is simply that participants received more training in Experiment 1 than Experiment 2 (450 trials vs. 300) and effects of orthographic feedback may need a given amount of trials to emerge. Consistent with this, there was a significant interaction between condition and training block in Experiment 2, suggesting that orthographic feedback does affect performance by the third block of training. A more intriguing possibility is that the effect of feedback may be consolidated over multiple days, as has been shown previously (Banai \& Lavner, 2014).

Although consolidation is not necessary for this type of generalized perceptual learning, it is possible that consolidation enhances learning. The average amount of learning in the orthographic feedback groups in Experiment 1 was much larger than in Experiment 2 (27.5 percentage points compared to 10.2). Given the generalization, time-course of learning, and the differences between the experiments, we suggest that updated mappings between synthesized acoustics and known phonetic categories may be consolidated over time.

Exactly how this consolidation occurs is uncertain; however, top-down active processes play an important role in speech perception (Heald \& Nusbaum, 2014) and selective attention has been linked to learning synthetic speech (Francis et al., 2000; Francis, Kaganovich, \& Driscoll-Huber, 2008). All training conditions require alertness to the task (see Seitz \& Watanabe, 2005); however, even the exposure-only condition demonstrated learning, suggesting conscious executive 
attention is not required but does facilitate learning, as evidenced by the feedback benefit. Neural findings suggest attention enhances synchronization among cortical populations processing input (Womelsdorf \& Fries, 2007). Over multiple days of training, attention directed to correct mappings (facilitated by feedback) may result in consolidated generalized learning, a process that could explain the differences in the magnitude of learning between experiments.

In conclusion, we show that explicit feedback supports perceptual learning, allowing listeners to adjust mappings between acoustic features of synthetic speech and phonetic representations, whereas incidental exposure produces only limited learning. Interestingly, reporting perceived lexical identity does not affect learning. This generalized learning may reflect consolidation of attention-mediated changes in acoustic-lexical mappings, providing insight into how acoustic constituents are combined to modify speech categories during learning.

\section{References}

Banai, K., \& Lavner, Y. (2014). The effects of training length on the perceptual learning of time-compressed speech and its generalization. The Journal of the Acoustical Society of America, 136(4), 1908-1917.

Bradlow, A. R., \& Bent, T. (2008). Perceptual adaptation to non-native speech, 106(2), 707-729. https://doi.org/10.1016/j.cognition.2007. 04.005

Brown, M., \& Kuperberg, G. R. (2015). A Hierarchical Generative Framework of Language Processing: Linking Language Perception, Interpretation, and Production Abnormalities in Schizophrenia. Frontiers in Human Neuroscience, 9(e29315), 611-23. https://doi.org/10.3389/fnhum.2015.00643

Chomsky, N. (1965). Aspects of a Theory of Syntax. Cambridge, MA: MIT Press.

Clarke, C. M., \& Garrett, M. F. (2004). Rapid adaptation to foreignaccented English. The Journal of the Acoustical Society of America, 116(6), 3647-3658. https://doi.org/10.1121/1.1815131

Davis, M. H., Johnsrude, I. S., Hervais-Adelman, A., Taylor, K., \& McGettigan, C. (2005). Lexical Information Drives Perceptual Learning of Distorted Speech: Evidence From the Comprehension of Noise-Vocoded Sentences. Journal of Experimental Psychology: General, 134(2), 222-241. https://doi.org/10.1037/0096-3445.134. 2.222

Demetras, M. J., Post, K. N., \& Snow, C. E. (1986). Feedback to first language learners: The role of repetitions and clarification questions. Journal of Child Language, 13(2), 275-292.

Egan, J. P. (1948). Articulation testing methods. The Laryngoscope, 58(9), 955-991.

Fenn, K. M., Margoliash, D., \& Nusbaum, H. C. (2013). Sleep restores loss of generalized but not rote learning of synthetic speech. Cognition, 128(3), 280-286. https://doi.org/10.1016/j.cognition. 2013.04.007

Fenn, K. M., Nusbaum, H. C., \& Margoliash, D. (2003). Consolidation during sleep of perceptual learning of spoken language. Nature, 425(6958), 614-616. https://doi.org/10.1038/nature01951

Francis, A. L., Baldwin, K., \& Nusbaum, H. C. (2000). Effects of training on attention to acoustic cues. Perception \& Psychophysics, 62(8), 1668-1680. https://doi.org/10.3758/BF03212164
Francis, A. L., Kaganovich, N., \& Driscoll-Huber, C. (2008). Cuespecific effects of categorization training on the relative weighting of acoustic cues to consonant voicing in English. The Journal of the Acoustical Society of America, 124(2), 1234. https://doi.org/10. $1121 / 1.2945161$

Francis, A. L., Nusbaum, H. C., \& Fenn, K. (2007). Effects of training on the acoustic phonetic representation of synthetic speech. Journal of Speech Language and Hearing Research, 50(6), 1445-1465. https:// doi.org/10.1044/1092-4388(2007/100)

Greenspan, S. L., Nusbaum, H. C., \& Pisoni, D. B. (1988). Perceptual learning of synthetic speech produced by rule. Journal of Experimental Psychology: Learning, Memory, and Cognition, 14(3), 421-433.

Guediche, S., Blumstein, S. E., Fiez, J., \& Holt, L. L. (2014). Speech perception under adverse conditions: insights from behavioral, computational, and neuroscience research. Frontiers in Systems Neuroscience, 7, 1-16. https://doi.org/10.3389/fnsys.2013.00126/ abstract

Guediche, S., Fiez, J. A., \& Holt, L. L. (2016). Adaptive Plasticity in Speech Perception: Effects of External Information and Internal Predictions. Journal of Experimental Psychology: Human Perception and Performance. https://doi.org/10.1037/xhp0000196

Heald, S. L. M., \& Nusbaum, H. C. (2014). Speech perception as an active cognitive process. Frontiers in Systems Neuroscience, 8, 35. https://doi.org/10.3389/fnsys.2014.00035

Hervais-Adelman, A., Davis, M. H., Johnsrude, I. S., \& Carlyon, R. P. (2008). Perceptual learning of noise vocoded words: Effects of feedback and lexicality. Journal of Experimental Psychology: Human Perception and Performance, 34(2), 460-474. https://doi.org/10. 1037/0096-1523.34.2.460

Idemaru, K., \& Holt, L. L. (2013). The developmental trajectory of children's perception and production of English /r/-/1/. The Journal of the Acoustical Society of America, 133(6), 4232. https://doi.org/10. 1121/1.4802905

Idemaru, K., \& Holt, L. L. (2014). Specificity of dimension-based statistical learning in word recognition. Journal of Experimental Psychology: Human Perception and Performance, 40(3), 10091021. https://doi.org/10.1037/a0035269

Keetels, M., Pecoraro, M., \& Vroomen, J. (2015). Recalibration of auditory phonemes by lipread speech is ear-specific, 141, 121-126. https://doi.org/10.1016/j.cognition.2015.04.019

Klatt, D. H. (1980). Software for a cascade/parallel formant synthesizer. The Journal of the Acoustical Society of America, 67(3), 971-995.

Kleinschmidt, D. F., \& Jaeger, T. F. (2015). Robust speech perception: Recognize the familiar, generalize to the similar, and adapt to the novel. Psychological Review, 122(2), 148-203. https://doi.org/10. 1037/a0038695

Kraljic, T., \& Samuel, A. G. (2006). Generalization in perceptual learning for speech. Psychonomic Bulletin \& Review, 13(2), 262-268. https:// doi.org/10.3758/BF03193841

Kuperberg, G. R., \& Jaeger, T. F. (2015). What do we mean by prediction in language comprehension? Language, Cognition and Neuroscience, 31(1), 32-59. https://doi.org/10.1080/23273798. 2015.1102299

Liu, R., \& Holt, L. L. (2015). Dimension-based statistical learning of vowels. Journal of Experimental Psychology: Human Perception and Performance, 41(6), 1783-1798. https://doi.org/10.1037/ xhp0000092

Loebach, J. L., Pisoni, D. B., \& Svirsky, M. A. (2010). Effects of semantic context and feedback on perceptual learning of speech processed through an acoustic simulation of a cochlear implant. Journal of Experimental Psychology: Human Perception and Performance, 36(1), 224-234. https://doi.org/10.1037/a0017609

Magnuson, J. S., Mirman, D., \& Harris, H. D. (2011). Computational Models of Spoken Word Recognition, 1-31. 
McClelland, J. L., Mirman, D., \& Holt, L. L. (2006). Are there interactive processes in speech perception? Trends in Cognitive Sciences, 10(8), 363-369. https://doi.org/10.1016/j.tics.2006.06.007

Mirman, D., McClelland, J. L., Holt, L. L., \& Magnuson, J. S. (2008). Effects of Attention on the Strength of Lexical Influences on Speech Perception: Behavioral Experiments and Computational Mechanisms. Cognitive Science, 32(2), 398-417. https://doi.org/ 10.1080/03640210701864063

Nittrouer, S., Lowenstein, J. H., \& Packer, R. R. (2009). Children discover the spectral skeletons in their native language before the amplitude envelopes. Journal of Experimental Psychology: Human Perception and Performance, 35(4), 1245-1253. https://doi.org/ 10.1037/a0015020

Norris, D., McQueen, J. M., \& Cutler, A. (2000). Merging information in speech recognition: Feedback is never necessary. Behavioral and Brain Sciences, 23(03), 299-325. https://doi.org/10.1017/ S0140525X00003241

Norris, D., McQueen, J. M., \& Cutler, A. (2003). Perceptual learning in speech. Cognitive Psychology, 47(2), 204-238. https://doi.org/10. 1016/S0010-0285(03)00006-9

Nusbaum, H. C., \& Pisoni, D. B. (1985). Constraints on the perception of synthetic speech generated by rule. Behavior Research Methods, Instruments, \& Computers, 17(2), 235-242.

Peelle, J. E., \& Wingfield, A. (2005). Dissociations in perceptual learning revealed by adult age differences in adaptation to time-compressed speech. Journal of Experimental Psychology: Human Perception and Performance, 31(6), 1315.

Pinker, S. (1994). The Language Instinct. New York, NY: William Morrow.
Schwab, E. C., Nusbaum, H. C., \& Pisoni, D. B. (1985). Some Effects of Training on the Perception of Synthetic Speech. Human Factors, 27(4), 395-408.

Seitz, A., \& Watanabe, T. (2005). A unified model for perceptual learning. Trends in Cognitive Sciences, 9(7), 329-334.

Sohoglu, E., \& Davis, M. H. (2016). Perceptual learning of degraded speech by minimizing prediction error. Proceedings of the National Academy of Sciences of the United States of America, 113(12), 201523266-10. https://doi.org/10.1073/pnas.1523266113

Vroomen, J., van Linden, S., de Gelder, B., \& Bertelson, P. (2007). Visual recalibration and selective adaptation in auditory-visual speech perception: Contrasting build-up courses. Neuropsychologia, 45(3), 572-577. https://doi.org/10.1016/j. neuropsychologia.2006.01.031

Womelsdorf, T., \& Fries, P. (2007). The role of neuronal synchronization in selective attention. Current Opinion in Neurobiology, 17(2), 154 160. https://doi.org/10.1016/j.conb.2007.02.002

Open Practices Statement The summary data are available at the following link: https://osf.io/xfv6k/?view_only= dcdb2b70806b4831b35f7cc3ed319abc and materials for the experiments are available on request. The experiment was not preregistered.

Publisher's note Springer Nature remains neutral with regard to jurisdictional claims in published maps and institutional affiliations. 